\title{
THREE THEOREMS ON FORM RINGS
}

\author{
LOUIS J. RATLIFF, JR.
}

\begin{abstract}
Three theorems concerning the form ring (= associated graded ring) $\mathbf{F}(R, I)$ of an ideal $I$ in a Noetherian ring $R$ are proved. The first characterizes, for a $P$-primary ideal in a locally quasi-unmixed ring, when $\mathbf{F}(R, I)_{\text {red }}$ is an integral domain in terms of when $\mathbf{F}\left(R_{P}, I R_{P}\right)_{\text {red }}$ is an integral domain. For an aribtrary Noetherian ring $R$ the second gives a somewhat similar characterization for $\mathbf{F}(R, J)$ to have only one prime divisor of zero for some ideal $J$ that is projectively equivalent to $I$. And the third characterizes unmixed semilocal rings in terms of the existence of an open ideal $I$ such that the zero ideal in $\mathbf{F}(R, I)$ is isobathy.
\end{abstract}

1. Introduction. If $I$ is an ideal in a Noetherian ring $R$ then the form ring $\mathbf{F}(R, I)$ of $R$ with respect to $I$ is the graded ring $R / I \oplus I / I^{2} \oplus I^{2} / I^{3} \oplus \cdots$. These rings have played an important role in many research problems in commutative algebra, so they have been deeply studied and many of their properties have been discovered. In this paper we consider two theorems concerning form rings that have previously appeared in the literature and a new third theorem. The first theorem gives a correct version of a result of Schenzel. It shows that, for locally quasi-unmixed Noetherian rings, $\mathbf{F}(R, I)_{\text {red }}$ is an integral domain if and only if $\mathbf{F}\left(R_{P}, I R_{P}\right)_{\text {red }}$ is an integral domain and $I$ has only one asymptotic prime divisor. The second theorem gives an analogous characterization for $\mathbf{F}(R, J)$ to have only one prime divisor of zero for some ideal $J$ that is projectively equivalent to $I$. And the third theorem shows that Nagata's form rings theorem, which gives a sufficient condition for a semilocal ring to be unmixed, actually characterizes unmixed semilocal rings.

2. Main theorems. In [1] Huneke proved several nice theorems concerning when, for a prime ideal $P$ in a Noetherian ring $R, \mathbf{F}(R, P)_{\text {red }}$ is an integral domain. These results placed some conditions on $R$ (such as being universally catenary and Nagata or Cohen-Macaulay) and usually showed that if $\mathbf{F}\left(R_{P}, P R_{P}\right)$ is an integral domain, then $\mathbf{F}(R, P)_{\text {red }}$ is an integral domain if and only if some other condition on $P$ (in terms of symbolic powers, analytic spread, etc.) holds. In [15, Theorem 3] Schenzel tried to prove a similar result applicable to all Noetherian rings, namely, $\mathbf{F}(R, P)_{\text {red }}$ is an integral domain if and only if $\mathbf{F}\left(R_{P}, P R_{P}\right)_{\text {red }}$ is an integral domain

Received by the editors July $1,1985$.

1980 Mathematics Subject Classification (1985 Revision). Primary 13A15; Secondary 13C99, 13H10.

Key words and phrases. Analytically unramified semilocal ring, asymptotic prime divisor, analytic spread of an ideal, essential prime divisor, form ring, integral closure of an ideal, isobathy ideal, Noetherian ring, normal ideal, projectively equivalent ideals, quasi-unmixed local ring, Rees ring, unmixed local ring, $u$-essential prime divisor.

Research on this paper was supported in part by the National Science Foundation, Grant MCS-8301248-02. 
and $\hat{A}^{*}(P)=\{P\}$. (Here, for an ideal $I$ in $R, \hat{A}^{*}(I)=\{Q \in \operatorname{Spec} R ; I \subseteq Q$ and $Q \in \operatorname{Ass}\left(R /\left(I^{n}\right)_{a}\right)$ for all large $\left.n\right\}$, where $\left(I^{n}\right)_{a}$ is the integral closure in $R$ of $I^{n}$.) This theorem would generalize and sharpen several results in $[\mathbf{1}]$, especially $[\mathbf{1}$, Theorem 2.2], but unfortunately the theorem does not hold in general; a specific counterexample can be given by considering $P=(z, z x) R$ in the ring $R$ of $[6$, Example 2, pp. 203-205] in the case $r=1$ and $m=0$. However, it follows from (2.1) that this theorem is true for primary ideals in locally quasi-unmixed Noetherian rings.

(2.1) THEOREM. Let $I$ be an ideal in a Noetherian ring $R$ and assume that $\operatorname{Rad}(I)=P$ is prime and that $R_{M}$ is quasi-unmixed for all maximal ideals $M$ in $R$ containing $I$. Then $\mathbf{F}(R, I)_{\text {red }}$ is an integral domain if and only if $\mathbf{F}\left(R_{P}, I R_{P}\right)_{\text {red }}$ is an integral domain and $\hat{A}^{*}(I)=\{P\}$.

ProOF. Let $\mathbf{R}=R[u, t I]$, where $t$ is an indeterminate and $u=1 / t$. Then in [13, Theorem 2.1] Rees showed that $\mathbf{F}(R, I) \cong \mathbf{R} / u \mathbf{R}$. (The restriction to the local ring case in $[\mathbf{1 3}]$ is not essential.) Also, $R_{P}\left[u, t I R_{P}\right] \cong \mathbf{R}_{R-P}$, so it suffices to show that $\operatorname{Rad}(u \mathbf{R})$ is prime if and only if $\operatorname{Rad}\left(u \mathbf{R}_{R-P}\right)$ is prime and $\hat{A}^{*}(I)=\{P\}$. For this, since $R_{M}$ is quasi-unmixed for all maximal ideals $M$ in $R$ containing $I$ and since $u \mathbf{R} \cap R=I$ it readily follows that $\mathbf{R}_{N}$ is quasi-unmixed for all maximal ideals $N$ in $\mathbf{R}$ containing $u \mathbf{R}$. Therefore, since $u$ is regular in $\mathbf{R}$ it follows from McAdam's Theorem [3, Proposition 4.1] that: (a) $\hat{A}^{*}(u \mathbf{R})=\{p \in \operatorname{Ass}(\mathbf{R} / u \mathbf{R})$; height $(p)=1\}$. Also, by [3, Proposition 3.18]: (b) $\hat{A}^{*}(I)=\left\{p \cap R ; p \in \hat{A}^{*}(u \mathbf{R})\right\}$. Therefore, if $\operatorname{Rad}(u \mathbf{R})=p$ is prime, then $\operatorname{Rad}\left(u \mathbf{R}_{R-P}\right)=p \mathbf{R}_{R-P}$ is prime and $p \cap R=P$, since $u \mathbf{R} \cap R=I$ and $\operatorname{Rad}(I)=P$, so $\hat{A}^{*}(I)=\{P\}$, by (a) and (b). And, conversely, if $\hat{A}^{*}(I)=\{P\}$ and $\operatorname{Rad}\left(u \mathbf{R}_{R-P}\right)=p \mathbf{R}_{R-P}$ is prime, then it readily follows from (a) and (b) that $\operatorname{Rad}(u \mathbf{R})=p$ is prime. Q.E.D.

Before proving a somewhat analogous result for arbitrary Noetherian rings, we first make several remarks concerning the condition $\hat{A}^{*}(I)=\{P\}$.

(2.2) REMARK. Let $I$ be an ideal in a Noetherian ring $R$. Then:

(2.2.1) It is shown in [3, Proposition 3.9] that the sets $\operatorname{Ass}\left(R /\left(I^{n}\right)_{a}\right)$ are monotonically increasing, so $\hat{A}^{*}(I)=\{P\}$ if and only if $\left(I^{n}\right)_{a}$ is $P$-primary for all $n \geq 1$.

(2.2.2) If $\hat{A}^{*}(I)=\{P\}$, then since minimal elements in $\operatorname{Ass}(\mathbf{R} / u \mathbf{R})$ are in $\hat{A}^{*}(u \mathbf{R})$ and $\hat{A}^{*}(I)=\left\{p \cap R ; p \in \hat{A}^{*}(u \mathbf{R})\right\}$ it follows that, for an arbitrary Noetherian ring $R, \mathbf{F}(R, I)_{\text {red }}$ is an integral domain if and only if $\mathbf{F}\left(R_{P}, I R_{P}\right)_{\text {red }}$ is. And since all minimal elements in $\operatorname{Ass}(\mathbf{R} / u \mathbf{R})$ are in $\hat{A}^{*}(u \mathbf{R})$ it also follows that $\mathbf{F}(R, I)$ has exactly $k$ minimal prime divisors of zero if and only if $\mathbf{F}\left(R_{P}, I R_{P}\right)$ does.

(2.2.3) It follows from McAdam's Theorem that if $R_{M}$ is quasi-unmixed for all maximal ideals $M$ in $R$ that contain $I$, then $\hat{A}^{*}(I)=\{P\}$ if and only if $l\left(I R_{Q}\right)<$ height $(Q)$ for all $Q \in \operatorname{Spec}(R)$ that properly contain $P$. (Here, $l\left(I R_{Q}\right)$ denotes the analytic spread of $I R_{Q}$.)

(2.2.4) If $I$ and $R$ are as in (2.1) and if $\mathbf{R}=R[u, t I]$, then the proof of (2.1) shows that the equivalent statements in $(2.1)$ are also equivalent to $\hat{A}^{*}(u \mathbf{R})=\{p\}$.

(2.2.5) It is shown in $[\mathbf{1 2},(2.2)]$ that if $\hat{A}^{*}(I)=\{P\}$, then $P \subseteq Q$ satisfy goingdown for all $Q \in \operatorname{Spec}(R)$ that contain $P$. Also, if for all maximal ideals $M$ in $R$ containing $I$ it holds that $R_{M}$ is unmixed, instead of just quasi-unmixed, then it is shown in $[\mathbf{1 1},(2.6)$ and $(2.2 .5)(\mathrm{f})]$ that if $I$ is $P$-primary, then $\hat{A}^{*}(I)=\{P\}$ is equivalent to: there exists $k \geq 1$ such that $I^{(n)} \subseteq I^{n-k}$ for all $n \geq k$, where 
$I^{(n)}=I^{n} R_{P} \cap R$ is the $n$th symbolic power of $I$. In particular, $I^{(n i)}=I^{(n) i}$ for all $n \geq k$ and for all $i \geq 1$, by $[\mathbf{1 1},(3.3)]$.

We now turn to the second theorem. For this theorem, recall that ideals $I$ and $J$ in a ring $R$ are projectively equivalent in case $\left(I^{i}\right)_{a}=\left(J^{j}\right)_{a}$ for some positive integers $i$ and $j$. Also recall that $E(I)=\{P \in \operatorname{Spec}(R) ; I \subseteq P$ and $(I+z) L$ is $P L$-primary for some $z \in \operatorname{Ass}(L)$, where $L$ is the completion of $\left.R_{P}\right\}$, and $U(I)=$ $\{p \cap R ; p \in E(u R[u, t I])\}$. (Elements in $E(I)$ (resp., $U(I))$ are called the essential (resp., u-essential) prime divisors of $I$; many properties of essential (resp., $u$ essential) prime divisors are proved in [4] (resp., [2]). In particular, $E(I) \cap \hat{A}^{*}(I) \subseteq$ $U(I) \subseteq \operatorname{Ass}\left(R / I^{n}\right)$ for all large $n$, by $[2,(2.3 .3)$ and (2.5.7)].)

(2.3) THEOREM. Let $I$ be an ideal in a Noetherian ring $R$ and assume that $\operatorname{Rad}(I)=P$ is prime. Then there exists an ideal $J$ in $R$ that is projectively equivalent to $I$ such that: $\operatorname{Ass}(\mathbf{F}(R, J))$ is a one-point set if and only if $\operatorname{Ass}\left(\mathbf{F}\left(R_{P}, J R_{P}\right)\right)$ is a one-point set and $U(J)=\{P\}$.

ProOF. Let $\mathbf{R}=R[u, t I]$, let $S$ be the set of regular elements in $\mathbf{R}-\bigcup\{p$; $p \in E(u \mathbf{R})\}$, and let $\mathbf{T}=\mathbf{R}_{S} \cap R[u, t]$. Then $\mathbf{T}$ is a finite $\mathbf{R}$-module, by [10, (3.3)], so $[10,(4.4)]$ shows that there exists a positive integer $k$ such that $u^{n} \mathbf{T} \cap R=$ $I^{n-k} J$ for all $n \geq k$, where $J=u^{k} \mathbf{T} \cap R$. Since $\mathbf{T}$ is a finite $\mathbf{R}$-module we have $I^{n}=u^{n} \mathbf{R} \cap R \subseteq u^{n} \mathbf{T} \cap R \subseteq u^{n} \mathbf{R}^{\prime} \cap R=\left(I^{n}\right)_{a}$ for all $n \geq 1$, where $\mathbf{R}^{\prime}$ is the integral closure of $\mathbf{R}$, so $I^{n} \subseteq I^{n-k} J \subseteq\left(I^{n}\right)_{a}$ for all $n \geq k$; hence $I$ and $J$ are projectively equivalent. Fix such an integer $k$ and ideal $J=u^{k} \mathbf{T} \cap R$ and let $\mathbf{A}=R[u, t J]$. Then $[10,(4.15)]$ shows that: (a) the maximal elements in $\operatorname{Ass}(\mathbf{A} / u \mathbf{A})$ are the maximal elements in $E(u \mathbf{A})$.

Now if $\operatorname{Ass}(\mathbf{F}(R, J))$ is a one-point set, then $\operatorname{Ass}(\mathbf{A} / u \mathbf{A})$ is, by [13, Theorem 2.1]. Therefore $U(J)=\{P\}$ (since it is clear that the minimal elements in $\operatorname{Ass}(\mathbf{A} / u \mathbf{A}$ ) are in $E(u \mathbf{A}))$ and $\operatorname{Ass}\left(\mathbf{A}_{R-P} / u \mathbf{A}_{R-P}\right)$ is a one-point set, so $\operatorname{Ass}\left(\mathbf{F}\left(R_{P}, J R_{P}\right)\right)$ is a one-point set, by $[\mathbf{1 3}$, Theorem 2.1], and $U(J)=\{P\}$. And, conversely, if $U(J)=\{P\}$ and $\operatorname{Ass}\left(\mathbf{F}\left(R_{P}, J R_{P}\right)\right)$ is a one-point set, then $\operatorname{Ass}\left(\mathbf{A}_{R-P} / u \mathbf{A}_{R-P}\right)$ is a one-point set (by $[\mathbf{1 3}$, Theorem 2.1]). Also, every maximal element in $\operatorname{Ass}(\mathbf{A} / u \mathbf{A})$ is in $E(u \mathbf{A})$, by (a), and every such element lies over $P$, since $U(J)=\{P\}$. Therefore, since $\operatorname{Ass}\left(\mathbf{A}_{R-P} / u \mathbf{A}_{R-P}\right)$ is a one-point set it follows that $\operatorname{Ass}(\mathbf{A} / u \mathbf{A})$ is a onepont set, so $\operatorname{Ass}(\mathbf{F}(R, J))$ is a one-point set. Q.E.D.

Concerning (2.3) it should be noted that if $I$ and $J$ are projectively equivalent ideals, then $U(I)=U(J)$, by $[\mathbf{2},(2.5 .6)]$, so the condition $U(J)=\{P\}$ in (2.3) is equivalent to the condition $U(I)=\{P\}$. Also, examples of primary ideals $I$ such that $U(I)=\{P\}$ were given in $[10,(4.5)]$. (2.4) has some additional remarks concerning the condition $U(I)=\{P\}$; the rings $\mathbf{T}$ and $\mathbf{A}$ in the proof of (2.3) will be used in some of these remarks.

(2.4) REMARK. (2.4.1) By (a) in the proof of (2.3) the maximal elements in $\operatorname{Ass}(\mathbf{A} / u \mathbf{A})$ are in $E(u \mathbf{A})$. Therefore it follows that if $U(I)=\{P\}$, then $u \mathbf{A}_{R-P} \cap$ $\mathbf{A}=u \mathbf{A}$, so $\mathbf{F}(R, J R)$ is a subring of $\mathbf{F}\left(R_{P}, J R_{P}\right)$ and these rings have the same total quotient rings. Therefore $\mathbf{F}(R, J)$ has only one (resp., only one minimal) prime divisor of zero if and only if $\mathbf{F}\left(R_{P}, J R_{P}\right)$ does.

(2.4.2) It is shown in $[2,(2.11)]$ that if the completion of $R_{M}$ has no imbedded prime divisors of zero for all maximal ideals $M$ in $R$ that contain $I$, then $U(I)=$ $\hat{A}^{*}(I)$. Therefore, for example, if $I$ is a primary ideal in an analytically unramified 
semilocal ring, then there exists an ideal $J$ that is projectively equivalent to $I$ such that: $\operatorname{Ass}(\mathbf{F}(R, J))$ is a one-point set if and only if $\operatorname{Ass}\left(\mathbf{F}\left(R_{P}, J R_{P}\right)\right)$ is a one-point set and $\hat{A}^{*}(J)=\{P\}$.

(2.4.3) It is shown in the proof of $[\mathbf{1 0},(4.15)]$ that there exists a one-to-one correspondence between $E(u \mathbf{T})$ and $E(u \mathbf{A})$ such that if $p^{\prime}$ and $p^{\prime \prime}$ correspond, then $p^{\prime} \cap R=p^{\prime \prime} \cap R$. And it is readily seen that $\operatorname{Ass}(\mathbf{T} / u \mathbf{T})=\left\{p \mathbf{R}_{S} \cap \mathbf{T} ; p \in \operatorname{Ass}(\mathbf{R} / u \mathbf{R})\right.$ and $p \cap S=\varnothing\}$, so there exists a one-to-one correspondence between $E(u \mathbf{R})$ and $E(u \mathbf{T})$, since the localization of an essential prime divisor is an essential prime divisor, by [4, (3.3.2)]. Therefore, since the minimal prime divisors of $u \mathbf{R}$ (resp., $u \mathbf{A}, u \mathbf{T})$ are in $E(u \mathbf{R})$ (resp., $E(u \mathbf{A}), E(u \mathbf{T})$ ) it follows that $\mathbf{F}(R, J)$ has exactly $k$ minimal prime divisors of zero if and only if $\mathbf{F}(R, I)$ does. In particular, it follows from (2.3) that if $\mathbf{F}\left(R_{P}, J R_{P}\right)_{\text {red }}$ is an integral domain and $U(J)=\{P\}$, then $U(I)=\{P\}$ (by projective equivalence) and $\mathbf{F}(R, I)_{\text {red }}$ is an integral domain.

(2.4.4) It was shown in [11] that if $I$ is $P$-primary, then $U(I)=\{P\}$ if and only if there exists $k \geq 1$ such that $I^{(n)} \subseteq I^{n-k} I^{(k)}$ for all $n \geq k$.

We now come to the third and final theorem, the second part of which is closely related to (2.3). For this result recall that an ideal $I$ in a ring $R$ is said to be isobathy in case every prime divisor $P$ of $I$ satisfies $\operatorname{depth}(P)=\operatorname{depth}(I)$. With this terminology, in [6, (25.1)] Nagata showed that if there exists an open ideal $I$ in a semilocal ring $R$ such that the zero ideal in $\mathbf{F}(R, I)$ is isobathy, then $R$ is unmixed. (2.5) shows that the converse of this also holds.

(2.5) THEOREM. A semilocal ring $R$ is unmixed if and only if there exists an open ideal $q$ in $R$ such that the zero ideal in $\mathbf{F}(R, q)$ is isobathy. If $R$ is unmixed and $I$ is any ideal in $R$, then there exists a positive integer $k$ and an ideal $J$ such that $I^{k} \subseteq J \subseteq\left(I^{k}\right)_{a}$ and the zero ideal in $\mathbf{F}\left(R, I^{i} J\right)$ is isobathy for all $i \geq 0$.

Proof. If the zero ideal in $\mathbf{F}(R, q)$ is isobathy, then $R$ is unmixed, by [6, (25.1)].

For the converse let $I$ be an ideal in $R$. It may clearly be assumed that $I \neq(0)$. Let $\mathbf{R}=R[u, t I]$ and $\mathbf{T}=\mathbf{R}_{S} \cap R[u, t]$, where $S$ is the set of regular elements in $\mathbf{R}-\bigcup\{p ; p \in E(u \mathbf{R})\}$. Then it follows as in the proof of $(2.3)$ that $\mathbf{T}$ is a finite $\mathbf{R}$-module, that there exists $k \geq 1$ such that $u^{n} \mathbf{T} \cap R=I^{n-k} J$ for all $n \geq k$, where $J=u^{k} \mathbf{T} \cap R$, and that $I$ and $J$ are projectively equivalent. Fix $i \geq 0$ and let $\mathbf{B}=R\left[u, t I^{i} J\right]$. Now $\mathbf{R}$ is locally unmixed, by [5, Corollary, p. 61], so $E(u \mathbf{R})=\{p \in \operatorname{Ass}(\mathbf{R} / u \mathbf{R}) ;$ height $(p)=1\}$, by $[\mathbf{2},(2.4)]$, so $[\mathbf{9},(8)]$ says that $u \mathbf{B}$ has no imbedded prime divisors, and so $\mathbf{F}\left(R, I^{i} J\right) \cong \mathbf{B} / u \mathbf{B}$ has no imbedded prime divisors of zero. Now, let $p$ be a (minimal) prime divisor of $u \mathbf{B}$, let $M$ be a maximal ideal in $R$ that contains $p \cap R$, and let $S=R-M$. Then $R_{S}\left[u, t I^{i} J R_{S}\right] \cong \mathbf{B}_{S}$ and $p \mathbf{B}_{S}$ is a height one prime divisor of $u \mathbf{B}_{S}$. Therefore, if $\mathbf{M}=\left(u, M, t I^{i} J\right) \mathbf{B}$, then $\operatorname{depth}\left(p \mathbf{B}_{S}\right)=\operatorname{height}\left(\mathbf{M B} \mathbf{B}_{S} / p \mathbf{B}_{S}\right)$, by $[\mathbf{8},(3.7)]$, and height $\left(\mathbf{M B} \mathbf{B}_{S} / p \mathbf{B}_{S}\right)=$ height $\left(\mathbf{M B}_{S}\right)-\mathbf{1}=\operatorname{height}(M)=\operatorname{altitude}(R)$, since $R$ and $\mathbf{B}_{\mathbf{M}}$ satisfy the first chain condition for prime ideals (since they are unmixed semilocal rings). Therefore it follows that $\operatorname{depth}(p)=\operatorname{altitude}(R)$, so the zero ideal in $\mathbf{F}\left(R, I^{i} J\right)$ is isobathy, since $\mathbf{F}\left(R, I^{i} J\right) \cong \mathbf{B} / u \mathbf{B}$. Finally, if $I$ is an open ideal, then so is $I^{i} J$. Q.E.D.

We next note two consequences of (2.5).

(2.6) REMARK. If $I$ is an ideal in an unmixed semi-local ring $R$, then:

(2.6.1) If $I$ is normal (that is, $\left(I^{n}\right)_{a}=I^{n}$ for all $n \geq 1$ ), then the zero ideal in $\mathbf{F}(R, I)$ is isobathy. 
(2.6.2) If $R$ is also analytically unramified, then the zero ideal in $\mathbf{F}\left(R,\left(I^{n}\right)_{a}\right)$ is isobathy for all large $n$.

PROOF. (2.6.1) If $I$ is normal, then the ring $\mathbf{T}$ in the proof of (2.5) is equal to $\mathbf{R}=R[u, t I]$, so $k=1$ and $J=I$, so the conclusion follows from (2.5) with $i=0$.

(2.6.2) If $R$ is analytically unramified, then $\left(I^{n}\right)_{a}$ is normal for all large $n$, by [14], so (2.6.2) follows from (2.6.1). Q.E.D.

We close with the following corollary of (2.5).

(2.7) COROLlary. A semilocal ring $R$ is unmixed if and only if for every ideal $I$ in $R$ there exists a projectively equivalent ideal $J$ in $R$ such that the zero ideal in $\mathbf{F}(R, J)$ is isobathy.

Proof. This is clear by (2.5). Q.E.D.

\section{REFERENCES}

1. C. Huneke, On the associated graded ring of an ideal, Illinois J. Math. 26 (1982), 121-137.

2. D. Katz and L. J. Ratliff, Jr., $U$-essential prime divisors and sequences over an ideal, Nagoya J. Math. 103 (1986), 39-66.

3. S. McAdam, Asymptotic prime divisors, Lecture Notes in Math., vol. no. 1023, Springer-Verlag, Berlin and New York, 1983.

4. S. McAdam and L. J. Ratliff, Jr., Essential sequences, J. Algebra 95 (1985), 271-235.

5. M. Nagata, On the chain problem of prime ideals, Nagoya Math. J. 10 (1956), 51-64.

6. __ Local rings, Interscience Tracts 13, Interscience, New York, 1962.

7. L. J. Ratliff, Jr., On prime divisors of the integral closure of a principal ideal, J. Reine Angew. Math. 255 (1972), 210-220.

8. __ On the prime divisors of zero in form rings, Pacific J. Math. 70 (1977), 489-517.

9. __ Powers of ideals in locally unmixed Noetherian rings, Pacific J. Math. 107 (1983), 459-472.

10. __ On linearly equivalent ideal topologies, J. Pure Appl. Algebra 41 (1986), 67-77.

11. $\ldots$, The topology determined by the symbolic powers of primary ideals, Comm. Algebra 13 (1985), 2073-2104.

12. _ Five notes on asymptotic prime divisors, Math. Z. 190 (1985), 567-581.

13. D. Rees, $A$ note on form rings and ideals, Mathematika 4 (1957), 51-60.

14. M. Sakuma and H. Okuyama, On a criterion for analytically unramification of a local ring, J. Gakugei Tokushima Univ. 15 (1966), 36-38.

15. P. Schenzel, Symbolic powers of prime ideals and their topology, Proc. Amer. Math. Soc. 93 (1985), 15-20.

Department of MATHEMAtics, UNiversity of CALIFornia, Riverside, CALiforNIA 92521 